FE DE ERRORES

\title{
Fe de errores a «Alargamiento de corona estético previo a rehabilitación protésica. Informe de caso»
}

\section{Erratum to «Aesthetic crown lengthening prior to prosthetic rehabilitation. Case report.»}

\author{
Alejandra Alvarado-Núñez ${ }^{1}$, Sonora Ramírez-Duarte ${ }^{2}$, Alejandro Nieto-Ramírez $^{3}$, René García-Contreras ${ }^{1 *}$ \\ 1. Escuela Nacional de Estudios Superiores, unidad \\ León, UNAM. León, México \\ 2. Escuela Nacional de Estudios Superiores, unidad \\ León, UNAM. León, México \\ 3. Universidad Nacional de Estudios Superiores, \\ unidad León. León, México \\ * Corresponding Author: René García-Contreras | \\ Escuela Nacional de Estudios Superiores (ENES) \\ Unidad León, UNAM. BIvd. UNAM 2011 Predio \\ El Sauce y el Potrero Comunidad Tepetates \\ C.P.37684 León, Gto. 7228856878 | E-mail: \\ Se hace constar que en el artículo publicado en su prestigiada revista denominado \\ Alargamiento de corona estético previo a rehabilitación protésica. Informe de caso por \\ Alejandra Alvarado-Núñez, Sonora Ramírez-Duarte, Alejandro Nieto-Ramírez, René \\ García-Contreras. Rev. Clin. Periodoncia Implantol. Rehabil. Oral Vol. 11(3); 170-172, 2018 \\ (DOI: 10.4067/S0719-01072018000300170) publicado en diciembre del 2018, se advierte \\ la omisión de la Esp. Trilce Melannie Virgilio Virgilio, quien colaboró con el autor Jaime \\ Alejandro Nieto-Ramírez en el desarrollo del caso clínico en la parte protésica. Dicha omisión \\ no fue intencional y por consenso de los autores se solicita la siguiente fe de erratas que \\ mencione de la siguiente manera: \\ "Los autores agradecen a Eduardo Dorado Suárez y a Trilce Melannie Virgilio Virgilio por \\ su cooperación en el desarrollo del caso clínico, facilitación de fotografías y elaboración del \\ tratamiento protésico".
} dentist.garcia@gmail.com

Trabajo recibido el 06/08/2017

Aprobado para su publicación el 07/12/2017

Véase contenido relacionado en DOI: 10.4067/S0719-01072018000300170 